\title{
Some subordination involving polynomials induced by lower triangular matrices
}

\author{
Saiful R. Mondal' , Kottakkaran Sooppy Nisar ${ }^{2}$ (D) and Thabet Abdeljawad ${ }^{3,4,5 *}$ (D)
}

\section{"Correspondence:}

tabdeljawad@psu.edu.sa

${ }^{3}$ Department of Mathematics and

General Sciences, Prince Sultan

University, 66833 Riyadh, Saudi

Arabia

${ }^{4}$ Department of Medical Research, China Medical University, 40402

Taichung, Taiwan

Full list of author information is

available at the end of the article

\begin{abstract}
The article considers several polynomials induced by admissible lower triangular matrices and studies their subordination properties. The concept generalizes the notion of stable functions in the unit disk. Several illustrative examples, including those related to the Cesàro mean, are discussed, and connections are made with earlier works.
\end{abstract}

MSC: Primary 30C45; secondary 40G05

Keywords: Polynomials; Admissible matrix; Stable functions; Cesàro means; Cesàro stable functions

\section{Introduction}

Let $\mathcal{A}$ and $\mathcal{A}_{1}$ denote the classes consisting of functions $\mathrm{h}$ in the unit disk $\mathbb{D}=\{w:|w|<1\}$ that are analytic and normalized by the conditions $\mathrm{h}(0)=0=\mathrm{h}^{\prime}(0)-1$ and $\mathrm{h}(0)=1$, respectively. Further let $\mathcal{S}^{*}\left(\alpha_{1}\right), \alpha_{1}<1$, be the class consisting of functions $\mathrm{h} \in \mathcal{A}$ starlike of order $\alpha_{1}$ satisfying $\operatorname{Re}\left(z \mathrm{~h}^{\prime}(z) / \mathrm{h}(z)\right)>\alpha_{1}, z \in \mathbb{D}$, and let $\mathcal{C}\left(\alpha_{1}\right)$ be the class of convex functions of order $\alpha_{1}$ with the characterization $\operatorname{Re}\left(1+z \mathrm{~h}^{\prime \prime}(z) / \mathrm{h}^{\prime}(z)\right)>\alpha_{1}, z \in \mathbb{D}$. It is evident that $f \in \mathcal{C}\left(\alpha_{1}\right)$ if and only if $z \mathrm{~h}^{\prime} \in \mathcal{S}^{*}\left(\alpha_{1}\right)$. The usual classes of starlike univalent functions and convex univalent functions will be denoted respectively by $\mathcal{S}^{*}:=\mathcal{S}^{*}(0)$ and $\mathcal{C}:=\mathcal{C}(0)$. Another important subclass is $\mathcal{K}\left(\alpha_{1}\right)$ consisting of $f \in \mathcal{A}$ close-to-convex of order $\alpha_{1}$ satisfying $\operatorname{Re}\left(z \mathrm{~h}^{\prime}(z) / g(z)\right)>0$ for some $g \in \mathcal{S}^{*}\left(\alpha_{1}\right)$. The class of close-to-convex univalent functions is denoted by $\mathcal{K}:=\mathcal{K}(0)$.

If $f$ and $g$ are analytic in $\mathbb{D}$, then $f$ is subordinate to $g$, written $f(z) \prec g(z)$, if there exists an analytic self-map $w$ in $\mathbb{D}$ satisfying $|w(z)| \leq|z|$ and $f(z)=g(w(z))$. For $f(z)=\sum_{k=0}^{\infty} a_{k} z^{k}$ and $g(z)=\sum_{k=0}^{\infty} b_{k} z^{k}$, the convolution (or Hadamard product) $f * g$ is given by the series $(f * g)(z)=\sum_{k=0}^{\infty} a_{k} b_{k} z^{k}$. The works of $[4,10,12,13,18]$ provide good resources on these subjects.

This paper studies stable functions, a notion first introduced by Ruscheweyh and Salinas [17]. Let $\mathbb{N}$ be the set of all positive integers, and $\mathbb{N}_{0}=\mathbb{N} \cup 0$. A function $h \in \mathcal{A}_{1}$ is $n$-stable with respect to $F \in \mathcal{A}_{1}$ if

$$
\frac{s_{n}(\mathrm{~h}, z)}{\mathrm{h}(z)} \prec \frac{1}{F(z)},
$$

(c) The Author(s) 2020. This article is licensed under a Creative Commons Attribution 4.0 International License, which permits use, sharing, adaptation, distribution and reproduction in any medium or format, as long as you give appropriate credit to the original author(s) and the source, provide a link to the Creative Commons licence, and indicate if changes were made. The images or other third party material in this article are included in the article's Creative Commons licence, unless indicated otherwise in a credit line to the material. If material is not included in the article's Creative Commons licence and your intended use is not permitted by statutory regulation or exceeds the permitted use, you will need to obtain permission directly from the copyright holder. To view a copy of this licence, visit http://creativecommons.org/licenses/by/4.0/. 
where $s_{n}(\mathrm{~h}, z)$ refers to the $n$th partial sum of the Taylor series of $\mathrm{h}$ about the origin. If a function $h$ is $n$-stable with respect to itself, then it is known as $n$-stable function, while $h$ is simply called stable (with respect to $F$ ) if $h$ is $n$-stable (with respect to $F$ ) for every $n \in \mathbb{N}_{0}$.

In [11], stable functions were extended to include the $(n, \beta)$-Cesàro stable functions. By introducing an admissible lower triangular matrix, this paper aims to generalize the notion of stable functions.

Let $n \in \mathbb{N}$ be fixed and $\mathcal{G}_{n}$ be a nonempty set consisting of $(n+1) \times(n+1)$ lower triangular matrices $G=\left(g_{r s}\right)$, where the entries $g_{r s} \geq 0, r, s=0,1, \ldots, n$, and satisfy the admissibility conditions:

(i) $g_{r 0}=1$ for every $r=0,1, \ldots, n$,

(ii) for each fixed $r \geq 1, g_{r s}=g_{r 1} g_{(r-1)(s-1)}, s=1, \ldots, n$,

(iii) for each fixed $r \geq 1,\left\{g_{r s}\right\}$ is a decreasing sequence.

The $(n+1)$ th row entities in the matrix $G$ induce a polynomial $P_{n}$ of degree $n$ given by

$$
P_{n}(z):=\sum_{k=0}^{n} g_{n k} z^{k}
$$

For any $\mathrm{h}(z)=\sum_{k=0}^{\infty} a_{k} z^{k} \in \mathcal{A}_{1}$, denote by $P_{n}(\mathrm{~h}, z)$ the polynomial

$$
P_{n}(\mathrm{~h}, z):=P_{n}(z) * \mathrm{~h}(z)=\sum_{k=0}^{n} g_{n k} a_{k} z^{k}
$$

It will be useful to rewrite (2) in the form

$$
P_{n}(\mathrm{~h}, z)=g_{n 1} P_{n-1}(\mathrm{~h}, z)+\sum_{k=0}^{n-1}\left(g_{n k}-g_{n 1} g_{n-1, k}\right) a_{k} z^{k}+g_{n n} a_{n} z^{n} .
$$

Here are some examples of admissible lower triangular matrices that will be used in the sequel.

Example 1.1 Let $G=\left(g_{i k}\right)$ be given by

$$
g_{i 0}=1 \quad \text { and } \quad g_{i k}= \begin{cases}1, & 1 \leq k \leq i \\ 0, & k \geq i+1\end{cases}
$$

If $\mathrm{h}(z)=\sum_{k=0}^{\infty} a_{k} z^{k}, a_{0}=1$, then

$$
s_{n}(\mathrm{~h}, z):=P_{n}(\mathrm{~h}, z)=P_{n}(z) * \mathrm{~h}(z)=\sum_{k=0}^{n} a_{k} z^{k}
$$

is the nth partial sum of the Taylor expansion of $f$ about the origin.

Example 1.2 For $\beta_{1} \geq 0$, let $G=\left(g_{i k}\right)$ be given by

$$
g_{i 0}=1 \quad \text { and } \quad g_{i k}= \begin{cases}\frac{\left(1+\beta_{1}\right)_{i-k}}{(i-k) !} \frac{i !}{\left(1+\beta_{1}\right)_{i}}, & 1 \leq k \leq i, \\ 0, & k \geq i+1 .\end{cases}
$$


Here $(a)_{l}$ denotes the Pochhammer symbol defined by $(a)_{0}=1$ and $(a)_{l}=a(a+1)_{l-1}, l \in \mathbb{N}$. If $\mathrm{h}(z)=\sum_{k=0}^{\infty} a_{k} z^{k}, a_{0}=1$, then

$$
\mathcal{S}_{n}^{\beta_{1}}(\mathrm{~h}, z):=P_{n}(\mathrm{~h}, z)=\sum_{k=0}^{n} \frac{\left(1+\beta_{1}\right)_{n-k}}{(n-k) !} \frac{n !}{\left(1+\beta_{1}\right)_{n}} a_{k} z^{k}
$$

is the nth Cesàro mean of order $\beta_{1}$ for $f$. When $\beta_{1}=0$, it reduces to the $n$th partial sum. The geometric properties of Cesàro mean can be seen in $[2,14,15]$.

The admissibility condition (iii) is satisfied since, for $1 \leq k \leq i$,

$$
\begin{aligned}
g_{i, k-1}-g_{i k} & =\frac{\left(1+\beta_{1}\right)_{i-k+1}}{(i-k+1) !} \frac{i !}{\left(1+\beta_{1}\right)_{i}}-\frac{\left(1+\beta_{1}\right)_{i-k}}{(i-k) !} \frac{i !}{\left(1+\beta_{1}\right)_{i}} \\
& =\frac{i !}{\left(1+\beta_{1}\right)_{i}} \frac{\left(1+\beta_{1}\right)_{i-k}}{(i-k) !}\left(\frac{i-k+\beta_{1}+1}{i-k+1}-1\right)=\frac{i !}{\left(1+\beta_{1}\right)_{i}} \frac{\left(\beta_{1}\right)_{i-k+1}}{(i-k+1) !} \geq 0 .
\end{aligned}
$$

Example 1.3 For $\beta \geq 0$, let $G=\left(g_{i k}\right)$ be given by

$$
g_{i 0}=1 \quad \text { and } \quad g_{i k}= \begin{cases}\left(1-\frac{k}{i+1}\right)^{\beta}, & 1 \leq k \leq i \\ 0, & k \geq i+1\end{cases}
$$

If $\mathrm{h}(z)=\sum_{k=0}^{\infty} a_{k} z^{k} \in \mathcal{A}_{1}$, then

$$
Q_{n}^{\beta}(\mathrm{h}, z):=P_{n}(\mathrm{~h}, z)=\sum_{k=0}^{n}\left(1-\frac{k}{n+1}\right)^{\beta} a_{k} z^{k} .
$$

Now the admissibility condition (iii) holds for $1 \leq k \leq i$ because

$$
\begin{aligned}
g_{i, k-1}-g_{i k} & =\left(1-\frac{k-1}{i+1}\right)^{\beta}-\left(1-\frac{k}{i+1}\right)^{\beta} \\
& =\frac{(i-k+2)^{\beta}-(i-k+1)^{\beta}}{(i+1)^{\beta}} \geq 0 .
\end{aligned}
$$

The polynomial $Q_{n}^{\beta}$ may also be expressed in terms of the Lerch transcendental function $\Phi(z, s, a)[1,5,9]$ given by the series

$$
\Phi(z, s, a)=\sum_{k=0}^{\infty} \frac{z^{k}}{(k+a)^{s}}
$$

with $|z|<1$, Res $>0$, and $a \in \mathbb{C} \backslash\{0,-1,-2, \ldots\}$. Thus

$$
Q_{n}^{\beta}(z)=\frac{(-1)^{\beta}}{(n+1)^{\beta}} \sum_{k=0}^{n} \frac{z^{k}}{(k-n-1)^{-\beta}}=\frac{(-1)^{\beta}}{(n+1)^{\beta}}\left(\Phi(z,-\beta,-n-1)-z^{n+1} \Phi(z,-\beta, 0)\right) .
$$

Example 1.4 For $\alpha_{1} \geq 0$, let $G=\left(g_{i k}\right)$ be given by

$$
g_{i 0}=1 \quad \text { and } \quad g_{i k}= \begin{cases}\frac{i+1+\delta}{i+1} \frac{i-k+1}{i-k+1+\delta}, & 1 \leq k \leq i \\ 0, & k \geq i+1\end{cases}
$$


If $f(z)=\sum_{k=0}^{\infty} a_{k} z^{k} \in \mathcal{A}_{1}$, then

$$
R_{n}^{\delta}(f, z):=P_{n}(f, z)=\sum_{k=0}^{n}\left(\frac{n+1+\delta}{n+1}\right)\left(\frac{n-k+1}{n-k+1+\delta}\right) a_{k} z^{k}
$$

The admissibility condition (iii) is easily seen to hold for $1 \leq k \leq i$ since

$$
\begin{aligned}
g_{i, k-1}-g_{i k} & =\frac{i+\delta+1}{i+1} \frac{i-k+2}{i-k+\delta+2}-\frac{i+\delta+1}{i+1} \frac{i-k+1}{i-k+\delta+1} \\
& =\frac{i+\delta+1}{i+1}\left(\frac{i-k+2}{i-k+\delta+2}-\frac{i-k+1}{i-k+\delta+1}\right) \\
& =\frac{\delta(i+\delta+1)}{(i+1)(i-k+\delta+2)(i-k+\delta+1)} \geq 0 .
\end{aligned}
$$

In terms of the Lerch transcendental function,

$$
\begin{aligned}
R_{n}^{\delta} & =\frac{n+1+\delta}{n+1} \sum_{k=0}^{n} \frac{n-k+1}{n-k+1+\delta} z^{k} \\
& =\frac{n+1+\delta}{n+1}\left(\frac{1-z^{n+1}}{1-z}+\delta \Phi(z, 1,-(n+1+\delta))-\delta z^{n+1} \Phi(z, 1,-\delta)\right) .
\end{aligned}
$$

Next consider the class $\mathcal{R}^{*}\left(\alpha_{1}\right)$ of prestarlike functions of order $\alpha_{1}, \alpha_{1}<1$, consisting of functions $f \in \mathcal{A}$ satisfying $f * k_{\alpha_{1}} \in \mathcal{S}^{*}\left(\alpha_{1}\right)$. Here $k_{\alpha_{1}}(z):=z /(1-z)^{2-2 \alpha_{1}}$ is an important member of the class $\mathcal{S}^{*}\left(\alpha_{1}\right)$. Evidently, $\mathcal{R}^{*}(1 / 2) \equiv \mathcal{S}^{*}(1 / 2)$ and $\mathcal{R}^{*}(0) \equiv \mathcal{C}$.

Another two functions of importance in the sequel are $f_{\mu}$ and $V_{\lambda, \alpha_{1}}$ given by

$$
f_{\mu}(z):=(1-z)^{-\mu}, \quad-1 \leq \mu \leq 1
$$

and

$$
\mathrm{V}_{\gamma, \beta}(z):=\left(\frac{1+(1-2 \beta) z}{1-z}\right)^{\gamma}, \quad 0<\gamma \leq 1,0 \leq \beta \leq 1,
$$

for $z \in \mathbb{D}$.

A brief computation shows that

$$
f_{\mu}-\frac{1-z}{\mu} f_{\mu}^{\prime}=0
$$

in $z \in \mathbb{D}$.

The article [3] discusses the stability of the function $\mathrm{V}_{\gamma, \beta}$ with respect to the function $f_{\gamma}(z)=(1-z)^{-\gamma}$. It is worth to mention here that for $\beta=1 / 2$ the stability of $\mathrm{v}_{\gamma, 1 / 2}$ with respect to $f_{\gamma}$ is equivalent to the stability (with itself) of $f_{\gamma}$. Thus, the result [3, Theorem 1] is another generalization of the stability of $f_{\lambda}$ proved in [17].

Definition 1.1 ( $P$-stable) Let $P_{n}$ be the polynomial given by $(1)$ which is induced by an admissible lower triangular matrix. A function $f \in \mathcal{A}_{1}$ is said to be $P_{n}$-stable with respect 
to $F \in \mathcal{A}_{1}$ if

$$
\frac{P_{n}(f, z)}{f(z)} \prec \frac{1}{F(z)}
$$

holds for a fixed $n \in \mathbb{N}$. In particular, $f$ will be called $P_{n}$-stable if it is $P_{n}$-stable with respect to itself. If $f$ is $P_{n}$-stable (with respect to $F$ ) for every $n \in \mathbb{N}_{0}$, then $f$ is simply said to be $P$-stable (with respect to $F$ ).

The article [19] also introduces a similar concept. In [19] the results encompass the generalization of the notion of the Cesàro stable functions, introduced in [11], by considering a generalized Cesàro operator

$$
\sigma_{n}^{b-1, c}(\mathrm{~h}, z)=\sigma_{n}^{b-1, c}(z) * \mathrm{~h}(z)
$$

where $\sigma_{n}^{b-1, c}$ is the $n$th Cesàro mean of order $(b-1, c)$. It is worth to mention here that $\sigma_{n}^{b-1, c}(z)$ can be induced through the admissible sequence

$$
g_{i 0}=1 \quad \text { and } \quad g_{i j}= \begin{cases}\frac{B_{i-j}}{B_{i},} & 1 \leq j \leq i \\ 0, & j \geq i+1,\end{cases}
$$

where

$$
B_{0}=1 \quad \text { and } \quad B_{i}=\frac{(b)_{i}}{(c)_{i}} \frac{1+b-c}{b}
$$

with $1+b>c>0$.

The next section presents the main results involving subordination of the polynomial $P_{n}\left(\mathrm{~V}_{\lambda, \beta}, z\right)=P_{n}(z) * \mathrm{~V}_{\lambda, \beta}(z)$. The results obtained will crystallize the notion of generalized stable functions. Using the polynomials induced by the admissible matrices given in Examples 1.1-1.4, the remaining sections will be devoted to the investigations of these generalized stable functions.

\section{The $P$-stability of $V_{\gamma, \beta}$}

The result in this section finds conditions that establish the $P$-stability of $\mathrm{V}_{\gamma, \beta}$ with respect to $(1-z)^{-\gamma}$ for $1 / 2 \leq \beta<1$ and $0<\gamma \leq 1$.

Recall a few basic properties and identities related to $\mathrm{V}_{\gamma, \beta}$ from the recent article [3]. The function $\mathrm{V}_{\gamma, \beta}$ can be represented by the series

$$
\mathrm{V}_{\gamma, \beta}(z)=: 1+\sum_{n=1}^{\infty} \mathrm{A}_{n}(\beta, \gamma) z^{n}
$$

with

$$
\mathrm{A}_{n}:=\mathrm{A}_{n}(\beta, \gamma)=\sum_{k=0}^{n} \frac{[\gamma]_{k}}{k !} \frac{(\gamma)_{n-k}}{(n-k) !}(1-2 \beta)^{k},
$$


where

$$
\begin{aligned}
& {[y]_{k}:=y(y-1)(y-2) \cdots(y-k+1),} \\
& (y)_{k}:=y(y+1)(y+2) \cdots(y+k-1),
\end{aligned}
$$

for $k=1,2, \ldots$ respectively and $[y]_{0}=1=(y)_{0}$.

Also, the function $\mathrm{V}_{\gamma, \beta}$ satisfies the differential equation

$$
\mathrm{V}_{\gamma, \beta}^{\prime}(z)-\frac{\gamma(2-2 \beta)}{1-2 \beta z-(1-2 \beta) z^{2}} \mathrm{~V}_{\gamma, \beta}(z)=0
$$

The basic properties of $A_{n}$ proved in [3] are summarized in the following lemma.

Lemma 2.1 Suppose that $0<\gamma \leq 1$ and $1 / 2 \leq \beta \leq 1$. Then

(a) $\mathrm{A}_{n} \geq 0$

(b) $(m+1)(n+1) \mathrm{A}_{n+1}-m n \mathrm{~A}_{n} \geq 0$,

for all $m, n \in \mathbb{N} \cup\{0\}$.

The $P$-stability of $\mathrm{V}_{\gamma, \beta}$ with respect to $(1-z)^{-\gamma}$ can be stated and proved as follows.

Theorem 2.2 Let $P_{n}$ be given by (1) with $g_{i 1} \leq 1$ for $i \geq 1, \mathrm{~V}_{\gamma, \beta}$ by (9), and $P_{n}\left(f_{\mu}, z\right)=P_{n}(z) *$ $\mathrm{V}_{\gamma, \beta}(z)$. For $0<\gamma \leq 1$ and $1 / 2 \leq \beta \leq 1$, the function $\mathrm{V}_{\gamma, \beta}$ is P-stable with respect to $f_{\gamma}(z)=$ $(1-z)^{-\gamma}$.

Proof It is enough to prove that

$$
\frac{(1-z)^{\gamma} P_{n}\left(\mathrm{~V}_{\gamma, \beta}, z\right)}{(1+(1-2 \beta) z)^{\gamma}} \prec(1-z)^{\gamma},
$$

which is equivalent to

$$
\frac{(1-z)\left(P_{n}\left(\mathrm{~V}_{\gamma, \beta}, z\right)\right)^{\frac{1}{\gamma}}}{1+(1-2 \beta) z} \prec(1-z)
$$

Define

$$
\begin{aligned}
& \mathrm{H}(z):=1-\frac{(1-z)\left(P_{n}\left(\mathrm{~V}_{\gamma, \beta}, z\right)\right)^{\frac{1}{\gamma}}}{1+(1-2 \beta) z} \\
& \mathrm{~K}_{1}(z):=\frac{(n+1) g_{n n} A_{n+1} z^{n}-\sum_{k=0}^{n-1}\left(g_{n(k+1)}-g_{n k}\right)(k+1) \mathrm{A}_{k+1} z^{k}}{(1+(1-2 \beta) z)^{2}}, \\
& \mathrm{~K}_{2}(z):=\frac{n g_{n n} A_{n} z^{n+1}+\sum_{k=2}^{n}\left(g_{n(k-1)}-g_{n k}\right)(k-1) \mathrm{A}_{k-1} z^{k}}{(1+(1-2 \beta) z)^{2}} .
\end{aligned}
$$

Note that $|\mathrm{H}(z)|<1$ is identical with the subordination given in (15), and to prove $|\mathrm{H}(z)|<1$, it is required to show that the Taylor series of $\mathrm{H}^{\prime}$ about the origin has nonnegative coefficients. 
A step by step computation yields the following identities:

$$
\left\{\begin{aligned}
P_{n}^{\prime}\left(\mathrm{V}_{\gamma, \beta}, z\right)= & P_{n}\left(\mathrm{~V}_{\gamma, \beta}^{\prime}, z\right)-(n+1) g_{n n} A_{n+1} z^{n} \\
& +\sum_{k=0}^{n-1}\left(g_{n(k+1)}-g_{n k}\right)(k+1) \mathrm{A}_{k+1} z^{k}, \\
z^{2} P_{n}^{\prime}\left(\mathrm{V}_{\gamma, \beta}, z\right)= & P_{n}\left(z \mathrm{~V}_{\gamma, \beta}^{\prime}, z\right), \\
P_{n}^{\prime}\left(z^{2} \mathrm{~V}_{\gamma, \beta}, z\right)= & P_{n}\left(z^{2} \mathrm{~V}_{\gamma, \beta}^{\prime}, z\right)+n g_{n n} A_{n} z^{n+1} \\
& +\sum_{k=2}^{n}\left(g_{n(k-1)}-g_{n k}\right)(k-1) \mathrm{A}_{k-1} z^{k}
\end{aligned}\right.
$$

Differentiation of (9) along with (18) gives

$$
\begin{aligned}
\mathrm{H}^{\prime}(z)= & \frac{(2-2 \beta) P_{n}\left(\mathrm{~V}_{\gamma, \beta}, z\right)}{(1+(1-2 \beta) z)^{2}}\left(P_{n}\left(\mathrm{~V}_{\gamma, \beta}, z\right)-\frac{(1-z)(1+(1-2 \beta) z)}{\gamma(2-2 \beta)} P_{n}^{\prime}\left(\mathrm{V}_{\gamma, \beta}, z\right)\right) \\
= & \frac{(2-2 \beta) P_{n}\left(\mathrm{~V}_{\gamma, \beta}, z\right)}{(1+(1-2 \beta) z)^{2}}\left[P_{n}\left(\mathrm{~V}_{\gamma, \beta}(z)-\frac{(1-z)(1+(1-2 \beta) z)}{\gamma(2-2 \beta)} \mathrm{V}_{\gamma, \beta}^{\prime}(z), z\right)\right. \\
& +(n+1) g_{n n} A_{n+1} z^{n}-\sum_{k=0}^{n-1}\left(g_{n(k+1)}-g_{n k}\right)(k+1) \mathrm{A}_{k+1} z^{k} \\
& \left.+(1-2 \beta)\left(n g_{n n} A_{n} z^{n+1}+\sum_{k=2}^{n}\left(g_{n(k-1)}-g_{n k}\right)(k-1) \mathrm{A}_{k-1} z^{k}\right)\right] \\
= & (2-2 \beta) P_{n}\left(\mathrm{~V}_{\gamma, \beta}, z\right)\left(\mathrm{K}_{1}(z)+(1-2 \beta) \mathrm{K}_{2}(z)\right) .
\end{aligned}
$$

Next we will investigate the nonnegativity of the coefficients of the Taylor series of

$$
\mathrm{K}_{1}(z)+(1-2 \beta) \mathrm{K}_{2}(z)
$$

about $z=0$. A series of calculation and careful arrangement of the terms leads to

$$
\begin{aligned}
\mathrm{K}_{1}(z) & +(1-2 \beta) \mathrm{K}_{2}(z) \\
= & \left((n+1) g_{n n} A_{n+1} z^{n}-\sum_{k=0}^{n-1}\left(g_{n(k+1)}-g_{n k}\right)(k+1) \mathrm{A}_{k+1} z^{k}\right)(1+(1-2 \beta) z)^{-2} \\
& +(1-2 \beta)\left(n g_{n n} A_{n} z^{n+1}+\sum_{k=2}^{n}\left(g_{n(k-1)}-g_{n k}\right)(k-1) \mathrm{A}_{k-1} z^{k}\right)(1+(1-2 \beta) z)^{-2} \\
= & g_{n n} z^{n}\left((n+1) A_{n+1}+(1-2 \beta) n A_{n} z\right)(1+(1-2 \beta) z)^{-2} \\
& +\left(\sum_{k=0}^{n-1}\left(g_{n k}-g_{n(k+1)}\right)(k+1) \mathrm{A}_{k+1} z^{k}+\sum_{k=2}^{n}\left(g_{n(k-1)}-g_{n k}\right)(k-1) \mathrm{A}_{k-1} z^{k}\right) \\
& \times(1+(1-2 \beta) z)^{-2} .
\end{aligned}
$$

Using Lemma 2.1, it is proved in [3] that the Taylor coefficients of

$$
\left((n+1) A_{n+1}+(1-2 \beta) n A_{n} z\right)(1+(1-2 \beta) z)^{-2}
$$


about $z=0$ are nonnegative. Consider the second expression

$$
\begin{aligned}
M= & \left(\sum_{k=0}^{n-1}\left(g_{n(k+1)}-g_{n k}\right)(k+1) \mathrm{A}_{k+1} z^{k}+(1-2 \beta) \sum_{k=2}^{n}\left(g_{n k}-g_{n(k-1)}\right)(k-1) \mathrm{A}_{k-1} z^{k}\right) \\
& \times(1+(1-2 \beta) z)^{-2} .
\end{aligned}
$$

A computation gives

$$
\begin{aligned}
M= & \left(\left(g_{n 0}-g_{n 1}\right) \mathrm{A}_{1}\right)(1+(1-2 \beta) z)^{-2} \\
& +\sum_{k=1}^{n-1}\left(g_{n k}-g_{n(k+1)}\right) z^{k}\left((k+1) \mathrm{A}_{k+1}+(1-2 \beta) k A_{k}\right)(1+(1-2 \beta) z)^{-2} \\
= & \left(\left(g_{n 0}-g_{n 1}\right) \mathrm{A}_{1}\right)\left(\sum_{m=0}^{\infty}(m+1)(2 \beta-1)^{m} z^{m}\right) \\
& +\sum_{k=1}^{n-1}\left(( g _ { n k } - g _ { n ( k + 1 ) } ) z ^ { k } \left((k+1) A_{k+1}\right.\right. \\
& \left.\left.+\sum_{m=0}^{\infty}\left((m+1)(k+1) \mathrm{A}_{k+1}-m k A_{k}\right)(2 \beta-1)^{m} z^{m}\right)\right) .
\end{aligned}
$$

Now by the definition of $\left\{g_{i j}\right\}$ we have $g_{n k} \geq g_{n(k+1)}$, and from Lemma 2.1, it follows that $(m+1)(k+1) A_{k+1}-m k A_{k} \geq 0$. Also, $1 / 2 \leq \beta \leq 1$ gives $2 \beta-1 \geq 0$. This concludes that all the coefficients of Taylor series of $\mathrm{K}_{1}(z)+(1-2 \beta) \mathrm{K}_{2}(z)$ are nonnegative. Consequently, the coefficients of Taylor series of $\mathrm{H}^{\prime}$ are nonnegative. Further, the nonnegativity of $\mathrm{A}_{n}$ implies that $\left|P_{n}\left(\mathrm{~V}_{\gamma, \beta}, z\right)\right| \leq P_{n}\left(\mathrm{~V}_{\gamma, \beta},|z|\right)$. Hence $\left|\mathrm{H}^{\prime}(z)\right| \leq \mathrm{H}(|z|)$.

Since $\mathrm{H}(0)=0$ and $\mathrm{H}(1)=1$, it follows that

$$
|\mathrm{H}(z)|=\left|\int_{0}^{z} \mathrm{H}^{\prime}(t) d t\right| \leq\left|\int_{0}^{1} \mathrm{H}^{\prime}(t z) d t\right| \leq \int_{0}^{1} \mathrm{H}^{\prime}(t) d t=1
$$

Therefore

$$
\left|1-\frac{(1-z)\left(P_{n}\left(\mathrm{~V}_{\gamma, \beta}, z\right)\right)^{\frac{1}{\gamma}}}{1+(1-2 \beta) z}\right|<1
$$

Equivalently,

$$
\frac{P_{n}\left(\mathrm{~V}_{\gamma, \beta}, z\right)}{\mathrm{V}_{\gamma, \beta}(z)} \prec(1-z)^{\gamma}
$$

for $n \in \mathbb{N}$. Hence the conclusion.

Choosing $\left\{g_{n k}\right\}$ as per Example 1.1, Theorem 2.2 reduces to the following result given in [3] on the stability of $\mathrm{V}_{\gamma, \beta}$.

Corollary ([3, Theorem 2.1]) For $0<\gamma \leq 1$ and $1 / 2 \leq \beta<1, \mathrm{~V}_{\gamma, \beta}$ is stable with respect to $f_{\gamma}(z)=1 /(1-z)^{\gamma}$. 
Consider $\left\{g_{n k}\right\}$ defined by Example 1.2, then Theorem 2.2 reduces to the following result which discusses the Cesàro stability of $\mathrm{V}_{\gamma, \beta}$.

Theorem 2.3 Suppose $\beta \geq 0$. The $0 \leq \gamma \leq 1$ and $1 / 2 \leq \beta \leq 1$, the function $\mathrm{V}_{\gamma, \beta}$ given by (9) is Cesàro stable with respect to $f_{\gamma}=(1-z)^{-\gamma}$.

Consider $\left\{g_{n k}\right\}$ as given in (11), then Theorem 2.2 can be restated as follows.

Theorem 2.4 Suppose $b \geq c>0$. The $0 \leq \gamma \leq 1$ and $1 / 2 \leq \beta \leq 1$, the function $\mathrm{V}_{\gamma, \beta}$ given by (9) is generalized Cesàro stable with respect to $f_{\gamma}=(1-z)^{-\gamma}$.

For $\beta=1 / 2$, we have $\mathrm{v}_{\gamma, 1 / 2}(z)=f_{\gamma}(z)=(1-z)^{-\gamma}$ and Theorem 2.4 reduces to [19, Theorem 2.1] for $\gamma \in[0,1]$.

\section{Conclusion and a future problem}

The polynomials induced by the lower triangular matrices play a vital role to generalize the main concept of stable functions which was defined based on subordination and the partial sum of the power series of the function. Notably, the immediate generalization of the partial sum of power series is the Cesàro sum, which is also induced in this article through a lower triangular matrix. It is evident from the articles $[6-8,11,16,17,19]$ that there is a strong relation between stable functions and trigonometric sums. Based on this, authors suggest investigating and developing some relevant trigonometric sums that can be induced by lower triangular matrices and can be interlinked with stable functions as future work. The extension of Theorem 2.2 for $\gamma \in[-1,0)$ is another open part that needs further investigation. Finally, the solution of the following problem may extend several ideas related to the stable functions.

Problem 3.1 Find the conditions on $\varrho$ and $\gamma$ so that

$$
\frac{(1-z)^{\varrho} P_{n}\left(\mathrm{~V}_{\gamma, \beta}, z\right)}{(1+(1-2 \beta) z)^{\varrho}} \prec(1-z)^{\varrho} .
$$

Acknowledgements

None.

\section{Funding}

The author T. Abdeljawad would like to thank Prince Sultan University for funding this work through research group Nonlinear Analysis Methods in Applied Mathematics (NAMAM) group number RG-DES-2017-01-17.

Availability of data and materials

Not applicable.

Competing interests

The authors declare that they have no competing interests.

Authors' contributions

The authors contributed equally and significantly in writing this paper. All authors read and approved the final manuscript.

\section{Author details}

'Department of Mathematics and Statistics, College of Science, King Faisal University, 31982 Al Ahsa, Saudi Arabia. ${ }^{2}$ Department of Mathematics, College of Arts and Sciences, Prince Sattam bin Abdulaziz University, 11991 Wadi Aldawasir, Saudi Arabia. ${ }^{3}$ Department of Mathematics and General Sciences, Prince Sultan University, 66833 Riyadh, Saudi Arabia. ${ }^{4}$ Department of Medical Research, China Medical University, 40402 Taichung, Taiwan. ${ }^{5}$ Department of Computer Science and Information Engineering, Asia University, Taichung, Taiwan. 


\section{Publisher's Note}

Springer Nature remains neutral with regard to jurisdictional claims in published maps and institutional affiliations.

Received: 27 July 2020 Accepted: 24 September 2020 Published online: 29 September 2020

\section{References}

1. Apostol, T.M.: Zeta and related functions. In: NIST Handbook of Mathematical Functions, pp. 601-616. U.S. Dept. Commerce, Washington (2010)

2. Bustoz, J.: Jacobi polynomial sums and univalent Cesàro means. Proc. Am. Math. Soc. 50, 259-264 (1975)

3. Chakraborty, S., Vasudevarao, A.: On stable functions. Comput. Methods Funct. Theory 18(4), 677-688 (2018)

4. Duren, P.L.: Univalent Functions. Springer, New York (1983)

5. Erdélyi, A.: Higher Transcendental Functions. Vols. I, II. McGraw-Hill, New York (1953)

6. Koumandos, S., Lamprecht, M.: On a conjecture for trigonometric sums and starlike functions, II. J. Approx. Theory $162(5), 1068-1084(2010)$

7. Koumandos, S., Ruscheweyh, S.: Positive Gegenbauer polynomial sums and applications to starlike functions. Constr. Approx. 23(2), 197-210 (2006)

8. Koumandos, S., Ruscheweyh, S.: On a conjecture for trigonometric sums and starlike functions. J. Approx. Theory 149(1), 42-58 (2007)

9. Lerch, M.: Note sur la fonction $\mathfrak{K}(W, x, s)=\sum_{k=0}^{\infty} \frac{e^{2 k \pi i x}}{\left(w w^{5}\right)^{5}}$. Acta Math. 11(1-4), 19-24 (1887)

10. Lewis, J.L.: Applications of a convolution theorem to Jacobi polynomials. SIAM J. Math. Anal. 10(6), 1110-1120 (1979)

11. Mondal, S.R., Swaminathan, A.: Stable functions and extension of Vietoris' theorem. Results Math. 62(1-2), 33-51 (2012)

12. Ruscheweyh, S.: Linear operators between classes of prestarlike functions. Comment. Math. Helv. 52(4), 497-509 (1977)

13. Ruscheweyh, S.: Convolutions in Geometric Function Theory. Presses Univ. Montréal, Montréal (1982)

14. Ruscheweyh, S.: Geometric properties of the Cesàro means. Results Math. 22(3-4), 739-748 (1992)

15. Ruscheweyh, S., Salinas, L.: Subordination by Cesàro means. Complex Var. Theory Appl. 21(3-4), 279-285 (1993)

16. Ruscheweyh, S., Salinas, L.: On starlike functions of order $\lambda \in[1 / 2,1)$. Ann. Univ. Mariae Curie-Skłodowska, Sect. A 54 $117-123(2000)$

17. Ruscheweyh, S., Salinas, L.: Stable functions and Vietoris' theorem. J. Math. Anal. Appl. 291(2), 596-604 (2004)

18. Ruscheweyh, S., Sheil-Small, T.: Hadamard products of Schlicht functions and the Pólya-Schoenberg conjecture. Comment. Math. Helv. 48, 119-135 (1973)

19. Sangal, P., Swaminathan, A.: On generalized Cesàro stable functions. Math. Inequal. Appl. 22(1), 227-247 (2019)

\section{Submit your manuscript to a SpringerOpen ${ }^{\circ}$ journal and benefit from:}

- Convenient online submission

- Rigorous peer review

- Open access: articles freely available online

- High visibility within the field

- Retaining the copyright to your article

Submit your next manuscript at $\boldsymbol{~ s p r i n g e r o p e n . c o m ~}$ 